
Cahiers
de a Recherche
Fur les Droits
Fondamentaux

\section{Cahiers de la recherche sur les droits fondamentaux}

$14 \mid 2016$

Urbanisme et droits fondamentaux

\title{
Une vie au service des droits de l'homme
}

À propos de Mes raisons d'État de Louis Joinet

A Life-Time Serving the Cause of Human Rights: A Look at Mes raisons d'État

by Louis Joinet

\section{Christophe Otero}

\section{OpenEdition \\ Journals}

Édition électronique

URL : https://journals.openedition.org/crdf/597

DOI : $10.4000 /$ crdf.597

ISSN : 2264-1246

\section{Éditeur}

Presses universitaires de Caen

\section{Édition imprimée}

Date de publication : 1 novembre 2016

Pagination : 109-110

ISBN : 978-2-84133-838-2

ISSN : $1634-8842$

Référence électronique

Christophe Otero, "Une vie au service des droits de l'homme », Cahiers de la recherche sur les droits fondamentaux [En ligne], 14 | 2016, mis en ligne le 01 octobre 2019, consulté le 14 novembre 2022. URL : http://journals.openedition.org/crdf/597 ; DOI : https://doi.org/10.4000/crdf.597 


\title{
Une vie au service des droits de l'homme À propos de Mes raisons d'État de Louis Joinet
}

\author{
Christophe OTERO \\ Docteur en droit public de l'université de Rouen \\ Membre associé du Centre universitaire rouennais d'études juridiques (CUREJ, EA 4703)
}

Les Mémoires ${ }^{1}$ de Louis Joinet plus encore que celles d'un épris de justice, comme elles sont sous-titrées, révèlent l'incroyable chemin suivi par un homme porté et animé, dans tous ses combats, par un esprit de justice. L'on cherche fréquemment, lorsqu'ils ne les livrent pas d'eux-mêmes, les motivations des juges à embrasser une telle carrière qui les fait plonger dans une "misère absolue » ${ }^{2}$ tout en devant, concurremment, "assumer le poids d'un office ${ }^{3}$. Comme l'écrit l'auteur,

[...] être juge n'est pas simple. C'est même, pour les freudiens, une des tâches sociales «impossibles», avec celles de guérir et d'éduquer. Il y a les médecins et les professeurs: les uns, au moins pourront soigner; les autres pour une part, instruire; mais de toutes les fonctions de gouvernance d'État, celle de juger est certainement la plus «impossible». (p. 43)

L'engagement au service de la justice de l'auteur vient sans doute du souvenir visuel et vivace laissé par le sort de sa coiffeuse à Nevers tondue à la Libération et qui marqua profondément sa prime enfance. La justice n'est effectivement pas celle expéditive des procès iniques sur la place publique. Elle est, au contraire, celle garantissant les droits de l'homme dans toutes leurs composantes et c'est à cette noble cause que Louis Joinet a entièrement et pleinement consacré sa vie. Il a indubitablement marqué de son empreinte, celle d'une logique constante de protection des droits fondamentaux, tant les institutions nationales et internationales que toutes les organisations gouvernementales et les organisations non gouvernementales par lesquelles il est passé.

Sa vie est jalonnée de rencontres décisives avec des hommes destinés à occuper des hautes fonctions judiciaires, telles celle de Luis Moreno Ocampo, qui devint procureur à la Cour pénale internationale, celle d'Antonio Cassese, futur président du Tribunal pénal international pour l'ex-Yougoslavie, ou encore celle de Laity Kama, qui sera par la suite président du Tribunal pénal international pour le Rwanda. Son parcours est atypique: il commença assez singulièrement sa carrière comme éducateur de rue, l'un des premiers en France. Cette première expérience n'a pu qu'enrichir et aguerrir Louis Joinet avant d'affronter la difficile mission de juger. Par le dialogue et l'écoute, les éducateurs de rue essayent de comprendre, autant que faire se peut, les ressorts et les raisons des jeunes en difficultés. Comme il le confesse, ce fut un excellent exercice, pour le magistrat qu'il sera par la suite, que de ne pas juger trop vite les comportements des délinquants.

L'ouvrage narre chronologiquement les trois vies non pas successives mais toujours entremêlées de Louis Joinet: d'abord, les parcours imprévus d'un magistrat syndicaliste, ensuite, ses années à Matignon au service de cinq Premiers ministres socialistes et, enfin, l'onusien au long cours qu'il sera durant plus de trente ans. Pendant toute sa carrière, il passera, parmi ces différentes activités, des unes aux autres sans jamais vouloir en délaisser aucune, à tel point que le qualificatif d'ubiquiste lui est fréquemment attribué. Peut-être faut-il voir, dans les multiples fonctions de Louis Joinet, l'écho de la réponse audacieuse mais néanmoins prémonitoire qu'il fit au grand oral de l'École nationale de la magistrature (ENM), dont il sortit major en 1966. À la question posée:

«Quelle est, pour vous, la principale qualité d'un juge?» J'étais sûr de mon effet. Là où ils attendaient l'indépendance, l'impartialité ou autres principes «à la Montesquieu » que l'on se doit de réciter pour une réponse de bon aloi, j'ai répondu, après un temps de réflexion quelque peu appuyé: "À la lumière de mon peu d'expérience, je dirai que la principale qualité d'un juge, c'est l'allergie à la routine». (p. 48)

Ces Mémoires offrent une plongée dans les années de création du Syndicat de la magistrature - dont il fut l'un des membres fondateurs - et ses objectifs originels: appeler les juges à se réinventer en tant que tels et faire bouger un corps judiciaire tout entier. Pour Louis Joinet,

1. L. Joinet, Mes raisons d'État: mémoires d'un épris de justice, Paris, La Découverte, 2013. Les références entre parenthèses renvoient à cette édition.

2. D. Salas, Le courage de juger, entretien avec F. Niel, Montrouge, Bayard, 2014, p. 10.

3. Ibid., p. 11. 
«la crise de la justice devait être dénoncée, y compris "de l'intérieur", pour que le peuple en fasse son affaire et qu'il se dote, pour la résoudre, de magistrats plus réellement indépendants» (p. 63). Ainsi, le Syndicat obtient la fin de la notation des juges par les procureurs, mais aussi le rétablissement de l'assemblée générale des magistrats dans chaque tribunal. Une restauration qui permettra, près de quarante ans plus tard, et à la grande joie de l'auteur, qu'un grand vent de révolte traverse l'assemblée générale de la Cour de cassation pour s'opposer notamment à la menace de suppression du juge d'instruction.

Les années 1970 virent l'essor de l'informatique. Intéressé à ces questions, notamment par sa participation au rapport Tricot de 1975, Louis Joinet deviendra ainsi directeur de la Commission nationale de l'informatique et des libertés (CNIL). Il chercha, dans ce cadre, à y protéger les droits fondamentaux et notamment la vie privée des citoyens contre les risques de dérives d'un système de fichage à grande échelle et généralisé, conscient, à l'instar de Michel Foucault, des enjeux et des dangers que pose la constitution de fichiers informatiques potentiellement liberticides. À partir de 1981 et jusqu'en 1995, Louis Joinet devint conseiller pour les droits de l'homme et la justice. Au service de cinq Premiers ministres, il dut trouver des solutions juridiques mais surtout réalistes aux problèmes tant calédoniens que basques et mettre en musique la doctrine Mitterrand d'alors, refusant les extraditions des anciens activistes et terroristes italiens. Dans ces différentes situations posant toutes des questions ardues d'amnistie, Louis Joinet s'opposa à ce qu'il dénomme «l'intégrisme légaliste» (p. 235). Selon lui,

Cet intégrisme est dangereux quand il conforte l'immobilisme et tarit l'inventivité juridique, à orienter bien sûr vers des progrès de l'État de droit. Une inventivité qui me tient à cœur comme juriste et même comme «rêveur " prêt à se situer parfois en fonction d'une légalité future. L'intégrisme légaliste revient à se réclamer d'un droit hypostasié, coupé de toute réalité et sans recours face à des crises où toute justice menace de disparaître. (p. 235)

Dans ces différents cas, au nom d'une certaine conception des droits de l'homme, l'impérialisme politique de la raison d'État et l'impérialisme juridique de l'État de droit, loin de s'opposer radicalement, doivent au contraire s'alimenter réciproquement. Il s'agit de jeter les bases d'une réconciliation en devenir où le politique doit pouvoir, l'espace de cette réconciliation, s'affranchir du droit actuel et laisser la place à ce que l'auteur nomme, en écho au titre de ses Mémoires, ses raisons d'État «qui furent des raisons d'humanité, l'emportant sur les raisons de souveraineté» (p. 261) de sorte à mieux pouvoir revenir ensuite à l'État de droit.

La dernière partie de l'ouvrage revient sur la facette la plus connue de l'auteur, c'est-à-dire ses années onu- siennes. En effet, dès 1978, Louis Joinet deviendra expert indépendant à la sous-commission des droits de l'homme de l'ONU dans le cadre de laquelle il a montré un «acharnement à protéger les victimes, [et] à dénoncer toutes les formes d'impunité » ${ }^{4}$ comme le souligne le documentaire qui lui a été consacré. Il fut ainsi rapporteur spécial sur la question de l'impunité des auteurs des violations des droits de l'homme. Ce rapport de 1997, qui lui a permis de «condenser le combat d'une vie entière» (p.328), contient un ensemble de principes de la justice transitionnelle, communément appelés «principes Joinet». Ceux-ci, au nombre de quarante-deux, s'articulent autour de trois grands piliers à destination des victimes: le droit de savoir (ou droit à la vérité), le droit à la justice et le droit à réparation. Ce document sert toujours de référence pour les acteurs impliqués dans des processus d'accords de paix, de transition et de retour vers la démocratie au lendemain d'un régime autoritaire ou totalitaire. Les Argentins et les Chiliens, au sortir de leurs périodes de dictature, puis les Sud-Africains, à l'issue de l'apartheid, furent les pionniers de tels processus. Pour Louis Joinet,

[...] un procès, même symbolique, est l'occasion de faire ressortir pleinement et publiquement la vérité. Il permet aux victimes d'être reconnues en tant que telles et de rendre inacceptables le sentiment et la volonté d'impunité des bourreaux 5 .

Comme l'écrit le philosophe Pierre Bouretz, l'influence du procès dépasse, dans ces cas, sa fonction de premier niveau:

[...] les victimes ne demandent effectivement pas un châtiment, une punition, elles ne réclament pas une réparation; elles attendent la reconnaissance publique, la médiation du procès comme moyen de transformer l'individuellement ressenti en socialement arrivé, le vécu en discours ${ }^{6}$.

L'un des derniers combats de Louis Joinet au service des droits de l'homme fut la Convention internationale contre les disparitions forcées (2006) dont il est l'un des principaux artisans. Ces disparitions forcées, qu'interroge le droit de savoir, posent aux juridictions le difficile problème de l'écoulement du temps. L'auteur conclut ainsi que la

[...] justice moderne devra de moins en moins se laisser soumettre par le temps, au détriment des victimes de crimes qui font les frais de procédures interminables.

La justice devrait désormais dompter le temps. (p. 328)

Depuis que le $\mathrm{XX}^{\mathrm{e}}$ siècle a créé des crimes imprescriptibles, «il n'y a plus de raisons que la justice ne cherche pas à remonter le temps autant que nécessaire» (p. 328).

Cet ouvrage offre indéniablement au lecteur une vision non pas idéalisée de la fonction de juger et du rôle de l'ONU mais celle d'un homme de compromis. La justice tant nationale qu'internationale est ainsi une création continue, qu’à l'instar de Pénélope il ne faut jamais croire achevée.

4. Un certain Monsieur Joinet, documentaire réalisé par F. Vaillant, 2011.

5. Lutter contre l'impunité: dix questions pour comprendre et pour agir, L. Joinet (dir.), Paris, La Découverte, 2002, p. 25.

6. P. Bouretz, C. Leben, A. Finkielkraut, L. Joinet, D. Lochak, J.-M. Varaut, «La prescription: table ronde du vendredi 22 janvier 1999 ", Droits, $\mathrm{n}^{\mathrm{o}}$ 31, 2000, Prescription et imprescriptibilité, p. 53. 